Erschienen in: Lang, Ewald/Zifonun, Gisela (Hrsg.): Deutsch - typologisch.

Berlin, New York: de Gruyter, 1996. S. 7-15.

(Jahrbuch des Instituts für deutsche Sprache 1995)

DOI: https://doi.org/10.1515/9783110622522-002

\title{
EWALD LANG
}

\section{Das Deutsche im typologischen Spektrum}

\section{Einführung in den Band}

\section{Deutsch - typologisch. Warum und wozu?}

Nach einem Rundblick auf die Wissenschaftslandschaft in der Germanistik und in den sich zunehmend verzweigenden anderen sprachwissenschaftlichen Disziplinen erwies sich die Themenwahl zur Jahrestagung 1995 als ebenso naheliegend und einleuchtend in der Sache wie werbend und anregend in der Absicht. Das Deutsche ist vielfältig und gründlich unter kontrastiven Gesichtspunkten analysiert und dokumentiert worden, aber es war von germanistischer Seite her bislang kaum Gegenstand typologischer Betrachtung. Umgekehrt ist die typologische Forschung selbst eher am zwischensprachlichen Vergleich von ausgewählten Schlüsselphänomenen interessiert, so daB das Deutsche dort zwar häufig vorkommt, aber meist nur in selektiven Ausschnitten.

Angesichts dieser Sachlage haben sich Mitarbeiter des Instituts für deutsche Sprache (IDS) und des Berliner Forschungsschwerpunkts "Allgemeine Sprachwissenschaft, Typologie und Universalienforschung" $(\text { FAS })^{1}$ zusammengetan und den Versuch verabredet, unter dem Thema "Deutsch - typologisch" eine neue Sicht auf die Struktur des Deutschen insgesamt zu ermitteln und wenigstens in Ausschnitten auch zu vermitteln. In den Blick zu nehmen für das Tagungsprogramm und die hier abgedruckten Beiträge sind zunächst die Prämissen des Unternehmens, nämlich die Rahmenbedingungen, innerhalb derer die Beschreibung einer einzelnen Sprache und die typologische Betrachtungsweise von Sprachen als unterschiedliche Zugänge innerhalb eines durch Universalien definierten Konzepts von "Sprache" zueinander in Beziehung zu setzen sind. Es geht dabei vornehmlich um folgende intradisziplinäre und methodische Zusammenhänge, zu deren Bewußtmachung grundsätzlich jeder der Aufsätze auf seine eigene Weise beiträgt:

1 Der FAS ist eine 1992 auf Empfehlung des Wissenschaftsrats gegründete außeruniversitāre Forschungseinrichtung, die bis Ende 1995 von der Förderungsgesellschaft Wissenschaftliche Neuvorhaben $\mathrm{mbH}$ München, einer Tochter der Max-Planck-Gesellschaft, getragen wird und ab 1996 als geisteswissenschaftliches Zentrum (gemeinsam finanziert vorn Land Berlin und der DFG) weitergeführt wird. Der Name des FAS ist sein Programm; vgl. das Kurzportrāt im SPRACHREPORT 1/1995. 
(1) Typologie und Universalien stehen im Konnex. Die Universalien definieren zum einen den Bereich der invarianten, d.h. der notwendigen und der unmöglichen, zum anderen den Bereich der variierenden, d.h. der möglichen, aber nicht notwendigen Struktureigenschaften von "(natürlicher) Sprache" (in Abgrenzung gegen nicht-menschliche Kommunikationssysteme einerseits und künstliche Zeichensysteme andererseits). Aufgabe der Sprachtypologie ist es, den Variationsbereich von "Sprache” anhand der Vielfalt seiner Belegungen durch die auf der Erde existierenden und in stetem Wandel begriffenen Sprachen zu erforschen und daraus rekurrierende, die Struktur von Sprachen nach Typen sortierende Charakteristica zu gewinnen.

(2) Die typologischen Merkmale bilden ein Raster grammatischer Optionen, das die Struktur einer Sprache über verschiedene Ebenen hinweg bestimmt, was sich u.a. in der thematischen Gruppierung der Beiträge im Band manifestiert. Für jede Sprache kann daher letztlich so etwas wie ein typologisches Gesamtporträt entworfen werden. Dies für das Deutsche zu konturieren, ist der gemeinsame Nenner aller Beiträge, wenngleich die Ausmalung des Porträts noch lange Zeit beanspruchen dürfte. Aber schon mit dem heute verfügbaren Raster ist nicht nur jede einzelne Sprache für bestimmte Parameter oder Konstruktionstypen im Spektrum der Sprachen einzuordnen (die Beiträge des Bandes diskutieren gerade die hierfür nötigen Vergleichsinstanzen), es sind auch die diversen Ausprägungen einer Sprache - hier also der deutschen - im Variationsbereich ihrer Dialekte und historischen Stadien lokalisierbar (vgl. speziell dazu die Beiträge von K. Donhauser, U. Engel/E. Geller, W. U. Wurzel und $\mathrm{E}$. Hentschel).

(3) Das so verstandene typologische Raster bildet einen geeigneten Rah$m e n$, in den kontrastive Studien einzubetten sind (Grundsätzliches dazu im Beitrag von E. König, speziellere Analysen u.a. in den Beiträgen von S. Olsen, V. Ehrich, J. Grabowski/P. WeiB), aus dem praktische Folgerungen für Deutsch als Fremdsprache zu ziehen sind (hier sei auf einen etwa zeitgleich erscheinenden Sammelband verwiesen ${ }^{2}$ ) und mithilfe dessen gerade auch dem muttersprachlichen Germanisten im Studium und danach wichtige Einsichten zu vermitteln sind.

2 Handwerker, Brigitte (Hg.) (1995): Fremde Sprache Deutsch. Grammatische Beschreibung - Erwerbsverläufe - Lehrmethodik. Tübingen. (Tübinger Beiträge zur Linguistik 409). 


\section{Programm und Beiträge}

Themenwahl und Vortragsangebot der IDS-Jahrestagung 1995 verstanden sich somit als Plädoyer für die typologische Betrachtungsweise im Rahmen von (1) bis (3) - exemplifiziert anhand der deutschen Sprache, die zu den am intensivsten untersuchten und am ausführlichsten dokumentierten Sprachen zählt und die bei aller Vielfalt der Interessen und Aspekte alle Teilnehmer der Tagung oder Leser des Jahrbuchs als Gegenstand und Medium verbindet. Ziel der Tagung und der hier z.T. stark überarbeiteten Beiträge ist zum einen die Präsentation neuer Fakten über die Struktur und die Verwendung des Deutschen auf der deskriptiven Ebene, zum anderen und vor allem aber die Vorstellung einer Sehweise, die das Deutsche auf eine jeweilige Vergleichsebene mit anderen Sprachen bringt und somit seine spezifischen, seine typisierbaren und seine generellen Struktureigenschaften in ein neues Licht rückt.

Was damit beabsichtigt war und - wie der Band zeigt - in einem erheblichen Ausmaß auch erreicht wurde, ist die Erzeugung von Aha-Effekten. Für germanistische Muttersprachler erscheint bislang Selbstverständliches unter einer typologischen Perspektive heilsam verfremdet - man schaue nur in die Beiträge über Wortstellung (B. Haftka, C. Wilder), Pronomina (M. Starke, W. Abraham), Genus (K.-M. Köpcke/D. Zubin) oder Orthographie (P. Eisenberg). Für den Auslandsgermanisten läßt sich manche als "typisch Deutsch" beleumdete Spezialität dieser Sprache (Mark Twain ${ }^{3}$ läßt grüßen!) aus typologischer Sicht auf erhellende Weise einordnen - man lese etwa die Beiträge zu Satzklammer (U. Engel/E. Geller, J. O. Askedal), Partikelverben (S. Olsen) oder Fugenelementen (N. Fuhrhop). Dem Typologen bietet der hier unternommene Versuch, eine einzelne gut untersuchte Sprache auf allen Ebenen der Strukturbildung kritisch zu mustern, Einblicke in unbeachtet gebliebene Zusammenhänge zwischen den strukturellen Optionen, die das grammatische Porträt einer Sprache ausmachen - dazu mehr in Abschnitt 5.

Weil die hier in Auswahlen vorgeführte typologische Betrachtungsweise mit ihrer Fokussierung auf das Deutsche so viele Aspekte umfaßt und zugleich so viele in ihren Interessen durchaus unterschiedliche Adressaten erreichen soll, ist eine kurze Einführung in die Lektüre des Bandes wohl angebracht. Auf ein das Inhaltsverzeichnis mehr oder minder para-

3 Mark Twains berūhmte Satire "The awful German language/Die schreckliche deutsche Sprache" (etwa in der preiswerten Ausgabe des Verlags Sindlinger-Burchartz, Frickenhausen 1993) im Seminar referieren zu lassen, ist ein probates Mittel, um Studenten der Germanistik im AnschluB daran für die typologische Betrachtungsweise zu interessieren. 
phrasierendes Vorwort wird verzichtet zugunsten von Abstracts, die den Beiträgen vorangestellt sind. Die Gruppierung der Beiträge nach thematischen Schwerpunkten gibt gewiB eine erste Orientierung, dennoch ist die in herkömmlicher Terminologie formulierte sachbezogene Einteilung nur eine Zugangshilfe - die eigentlichen Meriten der typologischen $\mathrm{Be}-$ trachtungsweise liegen in der Diversifikation der Vergleichsinstanzen quer durch die Ebenen sprachlicher Strukturbildung und in der Vernetzung der dabei erhobenen Befunde.

Um dem vornehmlich mit dem Deutschen befaBten Leser die zahlreichen Facetten von „Deutsch - typologisch” zu erschlieBen, werden im folgenden anhand bestimmter Leitfragen einige denkbare Routen durch die Beiträge des Bandes empfohlen, die unterschiedlichen Zugangsinteressen entsprechen.

\section{Wie betreibt man Typologie?}

Jegliche Wissenschaft, also auch die Sprachtypologie, beginnt mit der Klassifizierung von Befunden, aber sie kann dabei nicht stehenbleiben. Das damit umrissene Problem betrifft in unserem Falle die Eruierung geeigneter Vergleichskriterien und die Interpretation der aus ihnen zu gewinnenden Resultate. Wir finden im Band Bemühungen zur Präzisierung von Vergleichskriterien, zur Erweiterung des Inventars der für den Vergleich herangezogenen Parameter und auch zur Validität der jeweils erhebbaren Befunde.

Mehrere Beiträge des Bandes diskutieren den wissenschaftshistorisch zu verzeichnenden Übergang von den klassischen pauschalen Typisierungen (etwa den primär morphologisch basierten Typzuweisungen "isolierend", "agglutinierend", "fusionierend/flektierend" oder den Greenbergschen "basic word order types" gemäß der Abfolge von Subjekt, Objekt, Verb) zu wesentlich feiner differenzierten und weiter verzweigten Vergleichsinstanzen: B. Comrie plädiert vor diesem Hintergrund für eine konstruktionsbezogene Typologie; W. U. Wurzel zeigt, daB die klassischen morphologischen Typzuweisungen aufs Deutsche nicht disjunkt anwendbar sind, sondern daB sich die Charakteristik der deutschen Flexion diachron und synchron gerade aus einer anteilig spezifizierbaren Mischung derselben ergibt. Die Beiträge zur Negation überprüfen Jespersens Zyklus als typologisches Kriterium und kommen ebenfalls zu nötigen Differenzierungen: etwa bezüglich der Interaktion von Negationsverstärkung und Wortstellungstypologie (B. Lenz) oder bezüglich der perspektivischen Verkürzung, die der Zyklus induziert, wenn man ihn als einfache Erklärungsschablone für die diachrone Syntax der Negation des Deutschen anlegt ( $K$. Donhauser). 
Die meisten Beiträge bringen Argumente für die Erweiterung und Komplettierung der $\mathbf{z}$ wischensprachlichen Vergleichsbasis und für die Diversifikation des Kriterieninventars, um so das in (2) erwähnte Raster von Optionen zu verfeinern. In der Tat ist die typologische Betrachtungsweise prinzipiell auf jeder Ebene grammatischer Strukturbildung anzuwenden, nur sind viele Domänen diesbezüglich noch wenig erschlossen.

Als wichtige Vorstöße in dieser Richtung sind die Beiträge zur Phonologie zu betrachten: T.A. Hall demonstriert die Rolle von Silbe vs. Morphem als Domänèn phonotaktischer Bedingungen, U. Kleinhenz diskutiert den Status der phonologischen Phrase, und K. Alter illustriert durch experimentelle Befunde den Zusammenhang von Akzentplacierung und prosodischer Phrasierung. Eines der zentralen Themen der theoretischen Diskussion der letzten Jahre ist die Argumentstruktur lexikalischer Einheiten, d.h. das lexikalisch verankerte Scharnier zwischen morphosyntaktischer und semantischer Strukturbildung. Die Aufsätze von B. Primus, S. Olsen und V. Ehrich belegen, daB und wie die Beschaffenheit der Argumentstruktur von Verben typologische Signifikanz gewinnt. Als Versuche, die typologische Vergleichsbasis um zunächst nur sehr indirekt erschließbare Domänen wie Lexikalisierung (was wird wie in einem Wort semantisch codiert?) und Wortfeldstruktur (wie ordnen sich die lexikalisierten Einheiten zu einem Feld?) zu erweitern, dürfen die Beiträge u.a. von V. Ehrich, J. Grabowski/P. Weiß und E. Lang gelten.

Typologie ist nicht einfach ein Schüttelsieb mit unterschiedlichen Durchlaßöffnungen, sondern eine ständige Bemühung um Strukturvergleiche auf einer zweiten Stufe, nämlich um die Sortierung von Übereinstimmungen und Differenzen, die man in der Strukturbildung der verglichenen Sprachen, also auf der ersten Stufe, ausfindig gemacht hat. Nicht jeder Unterschied zwischen zwei Sprachen ist als typologisch relevant qualifizierbar (wie B. Comrie zeigt) und nicht alle typologisch einschlägigen Parameter haben dasselbe Gewicht (wie u.a. W. U. Wurzel, B. Haftka, C. Platzack, Ö. Dahl, T.A. Hall, P. Eisenberg für ganz verschiedene Bereiche der Grammatik illustrieren). Daher partizipiert die typologische Betrachtungsweise nicht nur rezeptiv (im Sinne der Übernahme theoretischer Vorgaben als Vergleichsinstanzen), sondern in zunehmendem Maße auch aktiv an der linguistischen Theoriediskussion (im Sinne der crosslinguistischen Überprüfung theoretischer Konzepte auf ihre generelle Gültigkeit und ihre typologische Aussagefähigkeit hin). Auch dafür finden sich im Band deutliche Spuren, insonderheit in den Beiträgen zu V2 und Satzklammer sowie zur Phonologie.

Theoretische Kontroversen stehen hier nicht im Zentrum, deshalb sprechen wir auch modellneutral von "typologischer Betrachtungsweise". 
Aber wegen des in (1) erläuterten Zusammenhangs von Typologie und Universalien gilt grundsätzlich, daß jeder, der sich mit der linguistischen Beschreibung einer Sprache befaßt, möglicherweise intuitiv und unreflektiert, aber von der Sache her unabdingbar als Typologe agiert. In diesem Sinne will der Band den primär mit dem Deutschen befaBten Lesern nicht neue Vorgehensrezepte andienen, sondern ihnen eher verdeutlichen, was sie (zumindest implizit) ständig praktizieren. Auch dies ein wichtiger Aspekt des Vorhabens, eine neue Sicht zu vermitteln.

\section{Was heißt „Deutsch als Mischtyp”?}

Nicht allein in den entsprechend rubrizierten Beiträge von Ö. Dahl, J. O. Askedal und U. Engel/E. Geller, sondern in fast allen Beiträgen wird das Deutsche unter der jeweiligen typologischen Optik als „Mischtyp” ausgewiesen. Wer auf holzschnittartige Einteilungen à la "flektierend", „V1, V2, VLetzt" und "freie Wortfolge" gefaBt war, könnte angesichts der zahlreichen Auskünfte über den Mischcharakter des Deutschen am Nutzen der typologischen Betrachtungsweise zweifeln.

Nun, „Mischtyp” ist hier nicht etwa das Verlegenheitsetikett für die Resultate einer Analyse, die vor einer unübersichtlichen Gemengelage kapituliert hat, sondern "Mischtyp” ist eine nach Domänen und Parametern jeweils exakt ausbuchstabierte Charakterisierung. Angesichts der detailliert vorgeführten Befunde ist "Mischtyp" genau die zutreffende Bezeichnung: sie verrät die nötige Umsicht gegenüber vorschnellen Einordnungen, und sie umfaßt die erklärte Absicht zu weitergehender Differenzierung. Tatsächlich besteht der hauptsächliche Erkenntnisgewinn der Beiträge des Bandes gerade im differenzierten Nachweis für die folgende generelle Aussage: das Spezifische des Deutschen besteht gerade in seiner jeweiligen Mischcharakteristik. Dies ist alles andere als trivial. Denn es sind die unter dem Label "Mischtyp" anteilig spezifizierten Struktureigenschaften, die synchron den jeweiligen Platz des Deutschen im Spektrum der Sprachen zu bestimmen und diesen Platz diachron als Resultat von Sprachwandel zu erklären gestatten und so die Puzzlesteine liefern für ein schrittweise auszufertigendes Gesamtporträt. Zur Illustration drei Beispiele aus ganz unterschiedlichen Bereichen:

Syntax: Im Hinblick auf die klassischen "basic word order types" hat sich schon sehr früh die Diskussion am Deutschen als "Mischtyp” (Hauptsatz SVO, Nebensatz SOV) entzündet und mittlerweile eine ganze Forschungsrichtung zu „V2-Phänomenen” begründet. Die Lokalisierung von "Deutsch als Mischtyp" zwischen Verbzweit- und Verbletzt-Sprache in Termen neuester Theorieentwicklung (etwa im „minimalistischen Programm") bildet auch den Angelpunkt für mehrere in diesem Band vorge- 
legte Beiträge. B. Haftka nimmt gängige, aber widersprüchliche typologische Einordnungen des Deutschen zum Ausgangspunkt, um mit einigen wenigen Annahmen über "scrambling" und "checking" den Mischtyp so auszubuchstabieren: Deutsch ist eine abgeleitete V2-Sprache mit zugrundeliegender SOV-Reihenfolge und flexibler, aber keineswegs ungeregelter Wortfolge. Ebenso folgt C. Platzack in seinem Beitrag über die germanischen V2-Sprachen dem auch für das Deutsche gültigen SymmetriePrinzip, dab das finite Verb im Hauptsatz stets dieselbe Position einnimmt, sieht jedoch für die typologische Variation in der Vorfeldbesetzung andere Mechanismen vor. C. Wilder schlieBlich ordnet die sog. V2Effekte bezüglich der Grammatik der Ellipse (etwa Deutsch vs. Englisch) als Resultat unabhängiger einzelsprachlicher Optionen innerhalb von sprachübergreifend wirksamen Prinzipien der Ellipsen-Bildung ein.

Diachronie: Wenn, wie in (1) oben festgestellt, die Typologie die Aufgabe hat, den kontingenten Variationsbereich sprachlicher Ausdrucksbildung zwischen „Notwendig" und „Unmöglich" zu strukturieren, dann legt sie natürlich auch die jeweiligen Spielräume für historische Veränderungen fest. Nun aber ist die diachrone Abfolge der Zustände einer Sprache keineswegs einfach als sequentielle Rückprojektion ihrer synchronen Koordinaten in die Sprachgeschichte zu erfassen, und wieder ist es die ausbuchstabierte Charakterisierung als „Mischtyp”, die einem solchen KurzschluB vorbeugt, wie die Beiträge von W. U. Wurzel zur Flexion und K. Donhauser zur Negation verdeutlichen.

Wortfeldstruktur: Daß sich selbst in einem generell zum Grundwortschatz gehörigen und durch Universalien hinreichend prädeterminierten Wortfeld wie dem der Ausdrücke für räumliche Dimensionen Partitionstypen unterscheiden lassen, zeigt der Aufsatz von E. Lang. Wieder erscheint hierbei das Deutsche als anteilig spezifizierter "Mischtyp" zwischen proportionsbasierter und betrachterbasierter Dimensionszuweisung. Genau dies aber ist die Basis, von der aus sprachspezifische Mehrdeutigkeiten, Lücken und Inferenzen erklärt werden können. Hier zeigt sich erneut, daß die Charakteristik "Mischtyp" gekoppelt ist mit einer exakten Angabe der Ingredienzen.

Schieblich ist eine begründete Kennzeichnung als "Mischtyp” auch der geeignete Ausgangspunkt, um das Deutsche in areallinguistischen Bezügen (vgl. die Beiträge von Ö. Dahl und E. König) und Interferenzprozessen (dazu u.a. E. Lang und U. Engel/E. Geller) zu positionieren. 
5. Was bringt die Typologie für die Grammatikschreibung?

Eine deutsche Grammatik, die dem hier als Zielbild entworfenen typologischen Gesamtporträt die nötige Grundierung verleihen könnte, gibt es noch nicht und es wird sie, trotz einiger beeindruckender Leistungen $^{4}$ auf dem Wege dahin, in nächster Zeit kaum als einheitliches Opus zwischen zwei Buchdeckeln geben können. Der Grund ist ebenfalls aus dem vorliegenden Band ablesbar: in dem Maße, wie unsere differenzierte Kenntnis über die typologisch relevanten Vergleichsinstanzen wächst, wächst auch die Kenntnis unserer Unkenntnis: stets liegen Sondierungstiefe und Darstellungsbreite im Widerstreit, und kompromißlerische Gesamtbeschreibungen werden zu Recht gescheut. Der unmittelbare Wert der typologischen Betrachtungsweise für die Grammatikdarstellung beruht - jenseits der in Abschnitt 3 erwähnten kritischen Funktion für die Theoriebildung und den Modellvergleich - in der Sichtbarmachung von bislang wenig beachteten Zusammenhängen zwischen den Strukturbildungsdomänen, genauer: in der Einsicht in die Funktionsweise von sog. Schnittstellen und in der Aufdeckung von Korrelationen zwischen bestimmten Parameterwerten innerhalb einer Sprache als typologisches Indiz. Hier ein Beispiel für einen solchen Zusammenhang $\mathbf{z w i s c h e n ~ W o r t s t r u k t u r ~ u n d ~ P h r a s e n s t r u k t u r : ~}$

S. Olsen leitet den handfesten Unterschied zwischen höchst produktiver Präfixverb-Bildung im Deutschen und vergleichsweise marginaler im Englischen ebenso wie die Unterschiede in der Argumentvererbung bei ansonsten vergleichbaren Partikelverben aus den unterschiedlichen Entwicklungslinien ab, die die beiden Sprachen vom gemeinsamen germanischen SOV aus genommen haben (Mittelenglisch wird SVO, das Deutsche bewahrt SOV und nimmt SVO im Hauptsatz dazu), und aus den damit induzierten Differenzen in der Köpfigkeit (im Englischen sind Phrasen kopfinitial, Wortstrukturen kopffinal, im Deutschen harmoniert

4 Hier ist nicht der Ort, auf zwei ganz unterschiedliche jüngere Werke, deren Titel für sich selbst sprechen, genauer einzugehen, aber sie verdienen im vorliegenden Kontext vorrangige Erwähnung: Abraham, Werner (1995): Deutsche Syntax im Sprachenvergleich. Grundlegung einer typologischen Syntax des Deutschen. Tübingen. (= Studien zur deutschen Grammatik 41); Glinz, Hans (1994): Grammatiken im Vergleich. Deutsch - Französisch - Englisch - Latein. Formen - Bedeutungen - Verstehen. Tübingen. (= Reihe Germanistische Linguistik 136). Nicht explizit typologisch ist die von Gisela Zifonun, Ludger Hoffmann, Bruno Strecker u.a. im IdS gerade fertiggestellte zweibāndige "Grammatik der deutschen Sprache” (Berlin/New York 1996) ausgerichtet. Als breit angelegte Gesamtdarstellung trāgt sie allein schon durch ihre Detailliertheit dazu bei, das typologische Portrāt des Deutschen zu vervollständigen. 
die Form des Partikelverbs mit der Rechtsköpfigkeit der Wortstruktur). - AbschlieBend eine mögliche Leseroute durch den Band, wie sie sich aus dem Verfolg von Zusammenhängen ergeben kann:

Jeder, der Deutsch als Fremdsprache lernen muB, nimmt die Genuszuweisungen für die Nomina als arbiträre Liste wahr und - etwa im Vergleich zum Englischen - als zusätzlichen Lernaufwand in Kauf. Nun zeigt der Beitrag von K.-M. Köpcke/D. Zubin, daB die Genuszuweisung im Deutschen - wiederum in einem arbeitsteiligen Mischverfahren aus phonologischen, morphologischen und semantischen Prinzipien - durchaus motiviert ist. Von da aus ist es nur ein kleiner Schritt, um zu sehen, daB die unterschiedliche Realisierung der Kategorie Genus im Deutschen (wo es für alle Nomina obligat zu spezifizieren ist) gegenüber dem Englischen (wo nur im Pronominalsystem noch Reste erhalten sind) nicht einfach unter Morphologie abzubuchen ist, sondern z.B. via Kongruenz auch syntaktische Folgeerscheinungen zeitigt: im Deutschen und Englischen ist wegen neutralisierter Genusmarkierung im Plural zulässig meine Onkel und Tanten/my uncles and aunts, im Singular aber gestattet eben nur das Englische die entsprechende Ellipse: $m y$ aunt and uncle/ ${ }^{*}$ meine Tante und Onkel/*mein Onkel und Tante, und damit sind wir bei den von $\mathrm{C}$. Wilder diskutierten generellen Ellipse-Prinzipien mit sprachspezifischer Anwendungssteuerung. Die genusspezifizierten (Personal-)Pronomina er/sie/es im Deutschen und he/she/it im Englischen (und ihre Gegenstücke in vielen anderen Sprachen) zeigen indes noch eine weitere syntaktische Involvierung: sie zerfallen nach ihrem Verhalten bezüglich Topikalisierung, Koordinierbarkeit etc. in die syntaktisch definierten Subgruppen "stark - schwach - klitisch" (M. Starke) und determinieren gemäß dieser Differenzierung in erheblichem MaBe u.a. die möglichen Besetzungen des "Mittelfeldes" (W. Abraham). Pronominal mehrfach besetzte Mittelfelder hinwiederum fallen dadurch auf, daB gegenüber ihrer Besetzung mit vollen NP "die relative Abfolge der pronominalen Argumente eigenen Regeln zu folgen scheint" (B. Primus) ...

So kommt man zwanglos und gleich über mehrere Anmarschwege vom Genus zur Syntax. Kurzum: die typologische Betrachtungsweise fungiert für die Grammatikschreibung des Deutschen als ständiger Impulsgeber für die Unterscheidung von Zusammenhängen und Indikator für den $\mathrm{Zu}$ sammenhang von Unterschieden. 